\title{
INTERPOLATION AND FACTORIZATION OF OPERATORS
}

\author{
STEVEN BLOOM, ANITA TABACCO VIGNATI AND MARCO VIGNATI
}

(Communicated by Paul S. Muhly)

\begin{abstract}
We apply the complex method of interpolation to families of infinite-dimensional, separable Hilbert spaces, and obtain a detailed description of the structure of the interpolation spaces, by means of a unique "extremal" operator-valued, analytic function.

We use these interpolation techniques to give a different proof, under weaker assumptions, of a theorem of A. Devinatz concerning the factorization of positive, infinite-rank, operator-valued functions.
\end{abstract}

Introduction. In this paper we apply the complex method of interpolation for families of Banach spaces, introduced by R. Coifman, M. Cwikel, R. Rochberg, Y. Sagher and G. Weiss, to the case when the boundary spaces are infinite dimensional, separable Hilbert spaces.

We show that all the "good" properties true for families of finite-dimensional Banach spaces (see [CCRSW 1]) hold also in this case. It is possible to describe the Hilbert space structure of the interpolation spaces by means of a unique, analytic, operator-valued function. This provides a different proof of Devinatz' theorem for the factorization of positive operators.

In $\S 3$ we give two applications. The first is an interpolation theorem for analytic families of linear operators; the second is a characterization of the intermediate spaces when the boundary family is given by Schatten ideals of operators acting on a varying family of Hilbert spaces.

The results contained in this paper are part of the last author's doctoral dissertation [Vi]. We are very grateful to Professors Richard Rochberg and Guido Weiss for introducing us to the subject, and for the help given during the research at Washington University.

1. The construction of the interpolation spaces. Let $H$ be a separable Hilbert space, with inner product $\langle\cdot, \cdot\rangle_{H}$ and norm $\|\cdot\|_{H}$, and let $B(H)$ be the algebra of all bounded, linear maps from $H$ to itself.

If $D$ denotes the open unit disk in the complex plane, and $\partial D$ is its boundary, we let $M: \partial D \rightarrow B(H)$ be a weakly-measurable function (i.e. $\langle M(\cdot) x, y\rangle_{H}$ is measurable for every $x, y \in H$ ), taking values in the set of positive, invertible elements of $B(H)$. In the following we shall identify the sets $\partial D$ and $T=[0,2 \pi)$, and the points $\vartheta \in T$ and $e^{i \vartheta} \in \partial D$.

To each $\vartheta \in T$ we assign a Hilbert space $H_{e^{i \vartheta}}=H_{\vartheta}=\left(H,\langle\cdot, \cdot\rangle_{\vartheta}\right)$, where

$$
\langle x, y\rangle_{\vartheta} \equiv\left\langle M\left(e^{i \vartheta}\right) x, y\right\rangle_{H}, \quad x, y \in H .
$$

Received by the editors September 12, 1986.

1980 Mathematics Subject Classification (1985 Revision). Primary 47A30. 
The norm $|\cdot|_{\vartheta}$ of $H_{\vartheta}$ satisfies

$$
k_{1}(\vartheta)\|\cdot\|_{h} \leq|\cdot|_{\vartheta} \leq k_{2}(\vartheta)\|\cdot\|_{H},
$$

where $k_{1}(\vartheta)=\left\|M^{-1}\left(e^{i \vartheta}\right)\right\|^{-1 / 2}$ and $k_{2}(\vartheta)=\left\|M\left(e^{i \vartheta}\right)\right\|^{1 / 2}$. We shall assume

$$
\log ^{+}\|M\|, \log ^{+}\left\|M^{-1}\right\| \in L^{1}(\partial D) .
$$

By $\widehat{H}^{2}(D ; H)$ we denote the class of all functions $F: D \rightarrow H$ of the form $F(z)=$ $\sum_{n=0}^{\infty} h_{n} z^{n}$, where $h_{n} \in H, z \in D$, and $\sum_{n=0}^{\infty}\left\|h_{n}\right\|_{H}^{2}<\infty$.

In [Hof] it is shown that these functions are analytic, the nontangential boundary values $F\left(e^{i \vartheta}\right)$ exist a.e. on $\partial D$, and

$$
\begin{aligned}
\|F\|^{\wedge} & \equiv \operatorname{Sup}_{0 \leq r<1}\left[\int_{T}\left\|F\left(r e^{i \vartheta}\right)\right\|_{H}^{2} \frac{d \vartheta}{2 \pi}\right]^{1 / 2} \\
& =\left[\int_{T}\left\|F\left(e^{i \vartheta}\right)\right\|_{H}^{2} \frac{d \vartheta}{2 \pi}\right]^{1 / 2}<\infty
\end{aligned}
$$

The space $\left(\widehat{H}^{2}(D ; H),\|\cdot\|^{\wedge}\right)$ is a Hilbert space, with inner product given by

$$
\langle F, G\rangle_{\widehat{H}^{2}} \equiv \int_{T}\left\langle F\left(e^{i \vartheta}\right), G\left(e^{i \vartheta}\right)\right\rangle_{H} \frac{d \vartheta}{2 \pi} .
$$

If

$$
h_{z}(\vartheta)=\frac{1}{2 \pi} \frac{1+z e^{-i \vartheta}}{1-z e^{-i \vartheta}}
$$

we construct the outer function $W_{1}(z) \equiv \exp \int_{T} \log k_{1}(\vartheta) h_{z}(\vartheta) d \vartheta$, and then define the space $\widehat{H}_{1}^{2}(D ; H)$ of all the analytic functions $F: D \rightarrow H$ such that $\|F\|_{1}^{\wedge}=$ $\left\|W_{1} F\right\|^{\wedge}<\infty$. Every function in $\widehat{H}_{1}^{2}(D ; H)$ has nontangential boundary values a.e. on $\partial D$, and this allows us to define the space

$$
\widehat{H}_{\#}^{2}=\widehat{H}_{\#}^{2}(D ; H) \equiv\left\{F: D \rightarrow H ; F \in \widehat{H}_{1}^{2}(D ; H), \int_{T}\left|F\left(e^{i \vartheta}\right)\right|_{\vartheta}^{2} \frac{d \vartheta}{2 \pi}<\infty\right\} .
$$

For each $z \in D$ we introduce in $\widehat{H}_{\#}^{2}$ the inner product

$$
\langle F, G\rangle_{\widehat{H}_{\#, z}^{2}} \equiv \int_{T}\left\langle F\left(e^{i \vartheta}\right), G\left(e^{i \vartheta}\right)\right\rangle_{\vartheta} P_{z}(\vartheta) d \vartheta
$$

where $P_{z}(\vartheta)=\operatorname{Re} h_{z}(\vartheta)$. It is easily seen that each $\widehat{H}_{\#, z}^{2}=\left(\widehat{H}_{\#}^{2},\langle\cdot, \cdot\rangle_{\widehat{H}_{\#, z}^{2}}\right)$ is a Hilbert space, and we denote its norm by $\|\cdot\|_{z}$. Moreover, if $N_{z}$ denotes the subspace of $\widehat{H}_{\#, z}^{2}$ consisting of those functions vanishing at $z, N_{z}$ is a closed subspace of $\widehat{H}_{\#, z}^{2}$.

We can now define the interpolation space associated with the family $\left\{H_{\vartheta}\right\}$. At each $z \in D$ the space $H_{z}$ is defined to be the set of all the equivalence classes in $\widehat{H}_{\#, z}^{2} / N_{z}$, endowed with the coset norm $|\cdot|_{z}$. Every $H_{z}$ is then a Hilbert space.

An equivalence class $[\mathbf{F}]$ is characterized by the value $F(z) \in H$. Conversely, if $h \in H$, the function $\widehat{F}_{z, h}(w) \equiv W_{2}(z) h / W_{2}(w)$, where

$$
W_{2}(w) \equiv \exp \int_{T} \log k_{2}(\vartheta) h_{w}(\vartheta) d \vartheta
$$


belongs to $\widehat{H}_{\#, z}^{2}$ and has value $h$ at $w=z$. Thus, the elements of $H_{z}$ can be identified with those of $H$, and

$$
|h|_{z}=\operatorname{Inf}\left\{\|F\|_{z} ; F \in \widehat{H}_{\#, z}^{2}, F(z)=h\right\} .
$$

LEMMA 1.8. Every Hilbert space $H_{z}$ is equivalent to $H$, with

$$
\left|W_{1}(z)\right|\|\cdot\|_{H} \leq|\cdot|_{z} \leq\left|W_{2}(z)\right|\|\cdot\|_{H} .
$$

ProOF. The use of the function $\widehat{F}_{z, h}$ introduced above shows the second inequality. Indeed,

$$
\begin{aligned}
|h|_{z} & \leq\left\|\widehat{F}_{z, h}\right\|_{z}=\left(\int_{T}\left|\widehat{F}_{z, h}\left(e^{i \vartheta}\right)\right|_{\vartheta}^{2} P_{z}(\vartheta) d \vartheta\right)^{1 / 2} \\
& \leq\left|W_{2}(z)\right|\left(\int_{T} \frac{|h|_{\vartheta}^{2}}{\left|W_{2}\left(e^{i \vartheta}\right)\right|^{2}} P_{z}(\vartheta) d \vartheta\right)^{1 / 2} \\
& \leq\left|W_{2}(z)\right|\left(\int\|h\|_{H}^{2} P_{z}(\vartheta) d \vartheta\right)^{1 / 2}=\left|W_{2}(z)\right|\|h\|_{H} .
\end{aligned}
$$

In order to prove the first inequality we recall a result of E. Vesentini [Ves], stating that if $F: D \rightarrow H$ is analytic, then $\log \|F(\cdot)\|$ is subharmonic in $D$. Thus, for $F \in \widehat{H}_{\#, z}^{2}$,

$$
\begin{aligned}
\|F(z)\|_{H} & \leq \exp \int_{T} \log \left\|F\left(e^{i \vartheta}\right)\right\|_{H} P_{z}(\vartheta) d \vartheta \\
& \leq \exp \int_{T} \log \left[k_{1}^{-1}(\vartheta)\left|F\left(e^{i \vartheta}\right)\right|_{\vartheta}\right] P_{z}(\vartheta) d \vartheta \\
& =\left|W_{1}(z)\right|^{-1} \exp \int_{T} \log \left|F\left(e^{i \vartheta}\right)\right|_{\vartheta} P_{z}(\vartheta) d \vartheta \\
& \leq\left|W_{1}(z)\right|^{-1}\|F\|_{z},
\end{aligned}
$$

which proves the lemma.

The next result shows that more can be said about (1.7).

LEMMA 1.9. If $z \in D$ and $h \in H$, there exists a unique extremal function $G_{z, h} \in \widehat{H}_{\#, z}^{2}$ satisfying
(a) $G_{z, h}(z)=h$;
(b) $|h|_{z}=\|G\|_{z}=\left|G_{z, h}\left(e^{i \vartheta}\right)\right|_{\vartheta}$ a.e. on $\partial D$.

PROOF. The infimum in (1.7) is taken over all the elements $F$ in the equivalence class $\left[\widehat{F}_{z, h}\right]=\widehat{F}_{z, h}+N_{z}$. This is a closed, convex subset of $\widehat{H}_{\#, z}^{2}$, and thus it has a unique element $G_{z, h}$ with minimal norm. If $\varphi(w)=\exp \int_{T} \log \left|G_{z, h}\left(e^{i \vartheta}\right)\right|_{\vartheta} h_{w}(\vartheta) d \vartheta$ and $\psi(w)=\varphi(z) G_{z, h}(w) / \varphi(w)$, then $\psi \in \widehat{H}_{\#, z}^{2}, \psi(z)=h$ and

$$
\begin{aligned}
|h|_{z} & \leq\|\psi\|_{z}=\left(\int_{T}\left|\psi\left(e^{i \vartheta}\right)\right|_{\vartheta}^{2} P_{z}(\vartheta) d \vartheta\right)^{1 / 2} \\
& =|\varphi(z)|=\exp \int_{T} \log \left|G_{z, h}\left(e^{i \vartheta}\right)\right|_{\vartheta} P_{z}(\vartheta) d \vartheta \\
& \leq\left\|G_{z, h}\right\|_{z}=|h|_{z} .
\end{aligned}
$$


Thus $|h|_{z}=\|\psi\|_{z}$; the uniqueness of the extremal function implies that $G_{z, h}(w)=$ $\psi(w)$ in $D$, and so $\left|G_{z, h}\left(e^{i \vartheta}\right)\right|_{\vartheta}=\left|\psi\left(e^{i \vartheta}\right)\right|_{\vartheta}=|\varphi(z)|=|h|_{z}$.

REMARK. Since $\widehat{H}_{\#, z}^{2}$ is a Hilbert space, the extremal function $G_{z, h}$ can be realized as the image $\Pi_{z}\left(\widehat{F}_{z, h}\right)$ of $\widehat{F}_{z, h}$ under the projection $\Pi_{z}$ of $\widehat{H}_{\#, z}^{2}$ onto the orthogonal complement $\left(N_{z}\right)^{\perp}$ of $N_{z}$.

THEOREM 1.10. There exists a unique operator-valued function $A: D \times D \rightarrow$ $B(H)$ such that $A(w, z) h=G_{z, h}(w) \forall w \in D$.

PROOF. The map $h \rightarrow G_{z, h}(w)$ is the composition of the map $h \rightarrow \widehat{F}_{z, h}$ and of $\Pi_{z}$. Since both are linear maps, the theorem is proved.

2. The Hilbert space structure of $H_{z}$. A more detailed study of the operator $A$ introduced in Theorem 1.10 will enable us to describe the structure of the spaces $H_{z}$.

LEMMA 2.1. If $z \in D,\left(N_{0}\right)^{\perp} \subset\left(N_{z}\right)^{\perp}$.

PROOF. Let $g \in N_{z}$, and define

$$
W_{1}(w) h(w)=\int_{T} W_{1}\left(e^{i \vartheta}\right) g\left(e^{i \vartheta}\right) P_{z}(\vartheta) P_{w}(\vartheta) d \vartheta
$$

In order to show that $h \in \widehat{H}_{1}^{2}(D ; H)$ we have only to prove that $W_{1} h$ is analytic. For $n$ a nonnegative integer we have

$$
\begin{aligned}
\left(W_{1} h\right)^{\wedge}(-n) & =\int_{T} W_{1}\left(e^{i \vartheta}\right) h\left(e^{i \vartheta}\right) e^{i n \vartheta} \frac{d \vartheta}{2 \pi} \\
& =\int_{T} W_{1}\left(e^{i \vartheta}\right) g\left(e^{i \vartheta}\right) P_{z}(\vartheta) e^{i n \vartheta} \frac{d \vartheta}{2 \pi} .
\end{aligned}
$$

Since $W_{1}(w) g(w)$ and $w^{n}$ are analytic, the last integral equals $(1 / 2 \pi) W_{1}(z) g(z) z^{n}=$ 0 . Thus $h \in \widehat{H}_{1}^{2}(D ; H)$, and since $h\left(e^{i \vartheta}\right)=g\left(e^{i \vartheta}\right) P_{z}(\vartheta)$ we obtain $h \in \widehat{H}_{\#, 0}^{2}$. Also,

$$
h(0)=\int_{T} h\left(e^{i \vartheta}\right) \frac{d \vartheta}{2 \pi}=\frac{g(z)}{2 \pi}=0
$$

and so $h \in N_{0}$. If $f \in\left(N_{0}\right)^{\perp}$, we have

$$
\langle f, g\rangle_{\widehat{H}_{\#, z}^{2}}=\int_{T}\left\langle f\left(e^{i \vartheta}\right), g\left(e^{i \vartheta}\right)\right\rangle_{\vartheta} P_{z}(\vartheta) d \vartheta=2 \pi\langle f, h\rangle_{\widehat{H}_{\#, 0}^{2}}=0 .
$$

Thus, $f \in\left(N_{z}\right)^{\perp}$ and the proof is complete.

THEOREM 2.2. If $z, w, \xi \in D$ :

(a) $A(z, w)=A(z, \xi) A(\xi, w)$;

(b) $A(z, w)=[A(w, z)]^{-1}$;

(c) $A(w, z)$ is analytic in both $w$ and $z$.

ProOF. (i) We first prove (a) for $w=0$. If $h \in H$ and $k=A(\xi, 0) h$, the extremal functions $G_{0, h}$ and $G_{\xi, k}$ coincides at $\xi$, and so their difference belongs to $N_{\xi}$. Also, $G_{0, h} \in\left(N_{0}\right)^{\perp} \subset\left(N_{\xi}\right)^{\perp}$ and $G_{\xi, k} \in\left(N_{\xi}\right)^{\perp}$. Thus $G_{0, h}(z)=G_{\xi, k}(z)$ in $D$, and this implies

$$
A(z, 0) h=G_{0, h}(z)=G_{\xi, k}(z)=A(z, \xi) k=A(z, \xi) A(\xi, 0) h
$$

which proves (i). 
(ii) $A(z, z)=I$ for every $z \in D$ follows from the definition of $A$.

(iii) Setting $z=0$ in (i) we get $I=A(0, \xi) A(\xi, 0)$.

(iv) Letting $S(\xi)=A(\xi, 0) A(0, \xi)$, it is immediate to see that every $S(\xi)$ is a projection. Moreover, if $S(\xi) h=0$, postmultiplication by $A(0, \xi)$ and (iii) show that $h=0$; thus, $S(\xi)=I$, which implies that $A(0, \xi)$ and $A^{-1}(\xi, 0)$ are inverses of each other.

(v) From (i) and (iv) it follows that $A(z, \xi)=A(z, 0) A(0, \xi)$.

(vi) From (v) and (iii) we obtain (a) for the general case.

(vii) Part (b) follows from (ii).

(viii) The analyticity of $A(w, z)=A(w, 0) A(0, z)$ with respect to $w$ follows from the definition of $A$. So, it is sufficient to show that $[A(z, 0)]^{-1}$ is analytic in a neighborhood of $z=0$. Obviously, $A(z, 0)=I+z B(z)$ where $B$ is analytic; the subharmonicity of $\left\|W_{1} G_{0, h}\right\|_{H}$ shows that $\|A(z, 0)\|_{B(H)} \leq c$ if $|z|<\varepsilon$, and so we can write $[A(z, 0)]^{-1}=I-z B(z)+z^{2} B^{2}(z)+\cdots$ which completes the proof.

We now set $A(z) \equiv A(z, 0)$, and this enables us to factor the function $A(w, z)$ as the product $A(w) A^{-1}(z)$ of two analytic factors, each one depending on only one variable, one the inverse of the other. This factorization is unique, up to right multiplication by a constant, invertible operator.

From Lemma 1.9(b) we have, for a.e. $\vartheta$,

$$
\begin{aligned}
|h|_{z}^{2} & =\left|A\left(e^{i \vartheta}, z\right) h\right|_{\vartheta}^{2} \\
& =\left\langle A\left(e^{i \vartheta}\right) A^{-1}(z) h, A\left(e^{i \vartheta}\right) A^{-1}(z) h\right\rangle_{\vartheta} \\
& =\left\langle M\left(e^{i \vartheta}\right) A\left(e^{i \vartheta}\right) A^{-1}(z) h, A\left(e^{i \vartheta}\right) A^{-1}(z) h\right\rangle_{H} \\
& =\left\langle\left[A^{*}\left(e^{i \vartheta}\right) M\left(e^{i \vartheta}\right) A\left(e^{i \vartheta}\right)\right] A^{-1}(z) h, A^{-1}(z) h\right\rangle_{H}
\end{aligned}
$$

which shows that $A^{*} M A$ is a positive operator, constant a.e. on $\partial D$. Setting $Q^{2}=A^{*} M A$, and denoting by $Q$ its unique positive square root, we obtain

$$
M\left(e^{i \vartheta}\right)=\left[Q A^{-1}\left(e^{i \vartheta}\right)\right]^{*}\left[Q A^{-1}\left(e^{i \vartheta}\right)\right] .
$$

REMARK. The existence of the (strong) nontangential limits $A\left(e^{i \vartheta}\right)$ and $A^{-1}\left(e^{i \vartheta}\right)$, and the fact that they are inverses of each other, are not obvious; we prefer to avoid entering into the details, which can be found in [Dev].

Letting $\phi(z) \equiv Q A^{-1}(z)$ we obtain

THEOREM 2.4. If $M: \partial D \rightarrow B(H)$ is weakly-measurable, with values in the set of positive, invertible operators, and $\log ^{+}\|m\|, \log ^{+}\left\|M^{-1}\right\|$ are integrable, there exists a unique $\phi: D \rightarrow B(H)$ such that:

(a) $\phi$ is analytic, invertible and $\phi^{-1}$ is analytic;

(b) $\lim _{z \rightarrow e^{i \vartheta}} \phi^{*}(z) \phi(z)=M\left(e^{i \vartheta}\right)$ a.e.;

(c) $\phi(0)>0$.

Moreover, if $z \in D$ and $h, k \in H$ :

(d) $\langle h, k\rangle_{z}=\langle\phi(z) h, \phi(z) k\rangle_{H}$;

(e) $|h|_{z}=\left\|\phi(z)_{h}\right\|_{H}$

(f) $\lim _{z \rightarrow e^{i \vartheta}}|h|_{z}=|h|_{\vartheta}$ a.e.

PrOOF. Having proved Theorem 2.2 and (2.3), we only have to show the uniqueness of $\phi$, and part (d). If $\psi(z)$ satisfies (a), (b), (c), let $U(z)=\psi(z) \phi^{-1}(z)$. Then $U$ is analytic, invertible and $U^{-1}$ is analytic. Moreover $U^{*}\left(e^{i \vartheta}\right) U\left(e^{i \vartheta}\right)=I$, and 
so $U\left(e^{i \vartheta}\right)$ is unitary a.e. on $\partial D$. We shall show that $U(z) \equiv I$. From the subharmonicity of $\log \|U(z)\|_{B(H)}$ and $\log \left\|U^{-1}(z)\right\|_{B(H)}$ it follows that $\|U(z)\|_{B(H)} \equiv 1$; thus $U(z)$ is unitary for every $z \in D$. Since $\psi(0)>0$, the uniqueness of its polar decomposition yields $U(0)=1$. For $x \in H$, let $f(z)=\langle U(z) x, x\rangle_{H} ; f$ is analytic in $D, f(0)=\|x\|_{H}^{2}$, and $\left|f\left(e^{i \vartheta}\right)\right| \leq\|x\|_{H}^{2}$. Thus $\langle U(z) x, x\rangle_{H}=f(z) \equiv\langle x, x\rangle_{H}$, and so $U(z) \equiv I$.

To prove (d) we recall that the spaces $H_{z}$ and $\widehat{H}_{\#, z}^{2} / N_{z}$ are isometric. Thus

$$
\begin{aligned}
\langle h, k\rangle_{z} & =\left\langle G_{z, h}, G_{z, k}\right\rangle_{\widehat{H}_{\#, z}^{2}} \\
& =\int_{T}\left\langle G_{z, h}\left(e^{i \vartheta}\right), G_{z, k}\left(e^{i \vartheta}\right)\right\rangle_{\vartheta} P_{z}(\vartheta) d \vartheta \\
& =\int_{T}\left\langle A^{*}\left(e^{i \vartheta}\right) M\left(e^{i \vartheta}\right) A\left(e^{i \vartheta}\right) A^{-1}(z) h, A^{-1}(z) k\right\rangle_{H} P_{z}(\vartheta) d \vartheta \\
& =\left\langle Q^{2} A^{-1}\left(z h, A^{-1}(z) k\right\rangle_{H}=\langle\phi(z) h, \phi(z) k\rangle_{H} . \quad\right.
\end{aligned}
$$

COROLlaRY 2.5. If $F: D \rightarrow H$ is analytic, then $\log |F(z)|_{z}$ is subharmonic in D.

ProOF. $|F(z)|_{z}=\|\phi(z) F(z)\|_{H}$, and we apply Vesentini's result.

We now extend the result obtained in Lemma 1.9(b) to the points in the interior of $D$.

LEMMA 2.6. Let $F: D \rightarrow H$ be analytic. Then $F(w)=A(w, z) F(z)$ for every $w, z \in D$ if and only if $|F(w)|_{w}=$ const in $D$.

PROOF. If $F(w)=A(w, z) F(z)$, then $F(w)=G_{z, F(z)}(w)$, and so

$$
\begin{aligned}
|F(w)|_{w} & =\left|G_{z, F(z)}(w)\right|_{w}=\left|A(w) A^{-1}(z) F(z)\right|_{w} \\
& =\left|\phi^{-1}(w) \phi(z) F(z)\right|_{w} \\
& =\|\phi(z) F(z)\|_{H}=|F(z)|_{z} \quad \text { for every } w \in D .
\end{aligned}
$$

Conversely, if $|F(w)|_{w}$ is constant in $D, F \in \widehat{H}_{\#, z}^{2}$ and $\|F\|_{z}=|F(z)|_{z}$. Thus $F$ is extremal for $F(z)$ at $w=z$, and the proof is complete.

REMARK. Let $\Omega$ be a simply connected domain, whose closure $\bar{\Omega}$ is contained in $D$, and whose boundary $\partial \Omega$ is a rectifiable, simple closed curve. The family $\left\{K_{\sigma}\right\}$, $\sigma \in \partial \Omega$, defined by $K_{\sigma}=H_{\sigma}$, is an interpolation family of Hilbert spaces (see Lemma 1.8). A natural question one can ask is what kind of relations there are between the spaces $K_{z}$ and $H_{z}, z \in \Omega$. A straightforward application of Lemma 2.6 then yields the Reiteration Property, i.e. $K_{z}=H_{z}$ for every $z \in \Omega$.

3. Applications. The first application that we describe involves families of linear operators. Let $\left\{H_{\vartheta}\right\}$ and $\left\{K_{\vartheta}\right\}$ be families of Hilbert spaces as those described in $\S 1$, generated by $H$ and $K$ respectively. Let $\left\{T_{w}\right\}, w \in \bar{D}$, be a family of linear operators from $H$ to $K$. We say that $\left\{T_{w}\right\}$ is an admissible analytic family if:

(i) for every $h \in H, T_{w} h$ is analytic in $D$ and $\lim _{z \rightarrow e^{i \varphi}} T_{w} h=T_{e^{i \varphi}} h$ a.e.;

(ii) $\left.\left|T_{e^{i \varphi}} h \|_{K_{\varphi}} \leq \eta\left(e^{i \varphi}\right)\right| h\right|_{H_{\varphi}}$ a.e. for every $h \in H$, and $\log ^{+} \eta \in L^{1}(\partial D)$.

THEOREM 3.1. If $\left\{T_{w}\right\}$ is an admissible analytic family of linear operators mapping $H$ into $K$, then $T_{w}: H \rightarrow K_{w}$ is continuous, and its norm is bounded by $\eta(w)=\exp \int_{T} \log \eta\left(e^{i \vartheta}\right) P_{w}(\vartheta) d \vartheta$ 
ProOf. Let $h \in H$ and $A(w, z) h$ be the extremal function of $h$ at $z \in D$, with respect to the family $\left\{H_{w}\right\}$. Since $T_{w}$ is linear, the function $w \rightarrow T_{w}(A(w, z))$ is analytic in $D$. So, using Corollary 2.5

$$
\begin{aligned}
\left|T_{z} h\right|_{K_{z}} & =\left|T_{z} A(z, z) h\right|_{K_{z}} \\
& \leq \exp \int_{T} \log \left|T_{e^{i \varphi}} A\left(e^{i \varphi}, z\right) h\right|_{K_{\varphi}} P_{z}(\varphi) d \varphi \\
& \leq \exp \int_{T} \log \left[\eta\left(e^{i \varphi}\right)\left|A\left(e^{i \varphi}, z\right) h\right|_{H_{\varphi}}\right] P_{z}(\varphi) d \varphi \\
& =\eta(z)|h|_{H_{z}} \cdot
\end{aligned}
$$

As a second application, we characterize the interpolation spaces obtained using the complex method for families of Banach spaces (see [CCRSW2]) when the boundary spaces are Schatten ideals associated with a family of varying Hilbert spaces $\left\{H_{\vartheta}\right\}$ of the type described above.

The interpolation spaces obtained from a family $\left\{A_{\vartheta}\right\}$ at $z \in D$ is denoted by $\left\{A_{\vartheta}\right\}[z]$. For the definition and the main properties of the Schatten ideals we refer to $[\mathbf{G K}]$.

By $N^{+}(D ; B(H))$ we denote the class of those functions $F: D \rightarrow B(H)$ such that $\langle F(z) x, y\rangle_{H} \in N^{+}(D)$ for every $x, y \in H$, where $N^{+}(D)$ denotes the "Nevanlinnaplus" class of functions on $D$ (see [Dur]).

THEOREM 3.2. If $p: \partial D \rightarrow[1, \infty]$ is measurable, and $\left\{H_{\vartheta}\right\}$ is the family of Hilbert spaces described by (1.1), then

$$
\left\{S_{p\left(e^{i \vartheta}\right)}\left(H_{\vartheta}\right)\right\}[z]=S_{p(z)}\left(H_{z}\right)
$$

where $1 / p(z)=\int_{T}\left[1 / p\left(e^{i \vartheta}\right)\right] P_{z}(\vartheta) d \vartheta$.

Proof. From Theorem 2.4 it follows that the sequence $\left\{\varphi_{j}\right\}_{1}^{\infty}$ is an orthonormal basis for $H$ if and only if $\left\{\varphi_{j}(z) \equiv\left\{\phi^{-1}(z) \varphi_{j}\right\}\right.$ is an orthonormal basis for $H_{z}$.

If $S \in B(H)$ is compact, it can be represented as $S=\sum_{j=1}^{\infty} s_{j}(S)\left\langle\cdot, \varphi_{j}\right\rangle_{H} \psi_{j}$, where $\left\{\varphi_{j}\right\},\left\{\psi_{j}\right\}$ are orthonormal sequences in $H$, and $\left\{s_{j}(S)\right\}$ is the sequence of the singular numbers of $S$. Then

$$
\phi^{-1}(z) S \phi(z)=\sum_{j=1}^{\infty} s_{j}(S)\left\langle\cdot, \varphi_{j}(z)\right\rangle_{z} \varphi_{j}(z)
$$

and the computation of $\left|\phi^{-1}(z) S \phi(z)\right|$ shows that the sequence of the singular numbers of $\phi^{-1} S \phi$ with respect to the space $H_{z}$ is still given by $\left\{s_{j}(S)\right\}$. Thus

$$
\|S\|_{S_{p}(H)}=\left\|\phi^{-1}(z) S \phi(z)\right\|_{S_{p}\left(H_{z}\right)} .
$$

First, we shall show that $\left\{S_{p\left(e^{i \vartheta}\right)}\left(H_{\vartheta}\right)\right\}[z] \rightarrow S_{p(z)}\left(H_{z}\right)$ is a norm-decreasing embedding. From the construction of the interpolation spaces (see [CCRSW2]), it is equivalent to showing that if $F \in N^{+}(D ; B(H)),\left\|F\left(e^{i \vartheta}\right)\right\|_{S_{p\left(e^{i \vartheta}\right)}}\left(H_{\vartheta}\right) \leq N\left(e^{i \vartheta}\right)$ a.e. and $\log ^{+} N \in L^{1}(\partial D)$, then $z \rightarrow \log \|F(z)\|_{S_{p(z)}\left(H_{z}\right)}$ is subharmonic in $D$.

If $p(z)$ is constant in $D,(3.3)$ and Vesentini's result imply that this is indeed the case. If $p(z)$ is not constant in $D$, we have $1<p(z)<\infty$ for every $z \in D$. Let $A$ be a finite-rank operator; we can find two orthonormal bases $\left\{\varphi_{j}\right\},\left\{\psi_{j}\right\}$ for $H$ such that $A=\sum_{j=1}^{n} s_{j}(A)\left\langle\cdot, \varphi_{j}\right\rangle_{H} \psi_{j}$. We also assume that $\|A\|_{S_{1}(H)}=\sum_{j=1}^{n} s_{j}(A)=1$. 
Let $z_{0} \in D, \rho\left\langle 1-\left|z_{0}\right|\right.$ and $z(\vartheta)=z_{0}+\rho e^{i \vartheta}$; we denote by $q(z)$ the conjugate index to $p(z)$, and by $a(z), b(z)$ the analytic functions whose real parts are $1 / p(z)$, $1 / q(z)$ respectively, and such that $a\left(z_{0}\right), b\left(z_{0}\right)$ are real numbers.

We define the operators

$$
A_{z}=\sum_{j=1}^{n} s_{j}(A)^{b(z)}\left\langle\cdot, \varphi_{j}\right\rangle_{H} \psi_{j}
$$

and

$$
A_{z}(z)=\phi^{-1}(z) \cdot A_{z} \cdot \phi(z)=\sum_{j=1}^{n} s_{j}(A)^{b(z)}\left\langle\cdot, \varphi_{j}(z)\right\rangle_{z} \psi_{j}(z)
$$

Then

$$
\left|A_{z}(z)\right|=\sum_{j=1}^{n} s_{j}(A)^{1-q(z)}\left\langle\cdot, \varphi_{j}(z)\right\rangle_{z} \varphi_{j}(z)
$$

and so

$$
\left\|A_{z}(z)\right\|_{S_{q(z)}\left(H_{z}\right)}=\left(\sum_{j=i}^{n} s_{j}(A)\right)^{1 / q(z)}=1
$$

If

$$
f(z)=\operatorname{Tr}_{H_{z}} F(z) \cdot A_{z}(z)=\sum_{j=1}^{\infty} s_{j}(A)^{b(z)}\left\langle\phi(z) \cdot F(z) \cdot \phi^{-1}(z) \psi_{j}, \varphi_{j}\right\rangle_{H}
$$

where $\operatorname{Tr} S$ denotes the trace of the operator $S$, then $f$ belongs to $N^{+}(D)$, and so $\log |f(z)|$ is subharmonic. This implies

$$
\begin{aligned}
\log \left|f\left(z_{0}\right)\right| & \leq \int_{T} \log |f(z(\vartheta))| \frac{d \vartheta}{2 \pi} \\
& =\int_{T} \log \left|\operatorname{Tr}_{H_{z(\vartheta)}} F(z(\vartheta)) \cdot A_{z(\vartheta)}(z(\vartheta))\right| \frac{d \vartheta}{2 \pi} \\
& \leq \int_{T} \log \left\|F(z(\vartheta)) A_{z(\vartheta)}(z(\vartheta))\right\|_{S_{1}\left(H_{z(\vartheta)}\right)} \frac{\partial \vartheta}{2 \pi} \\
& \leq \int_{T} \log \|F(z(\vartheta))\|_{S_{p(z(\vartheta))}\left(H_{z(\vartheta)}\right)} \frac{d \vartheta}{2 \pi} .
\end{aligned}
$$

We now take the supremum over all operators $A$, and the last inequality can be rewritten as

$$
\log \left\|F\left(z_{0}\right)\right\|_{S_{p\left(z_{0}\right)}\left(H_{z_{0}}\right)} \leq \int_{T} \log \left\|F\left(z_{0}+\rho e^{i \vartheta}\right)\right\|_{S_{p\left(z_{0}+\rho e^{i \vartheta}\right)}\left(H_{z_{0}+\rho e^{i \vartheta}}\right)} \frac{d \vartheta}{2 \pi},
$$

which proves the desired inclusion.

In order to show the second part of the theorem, we consider

$$
A=\sum_{j=1}^{n} s_{j}(A)\left\langle\cdot, \varphi_{j}\left(z_{0}\right)\right\rangle_{z_{0}} \psi_{j}\left(z_{0}\right)
$$


with $\|A\|_{S_{p(z)}\left(H_{z}\right)}=1$, where $\left\{\varphi_{j}\left(z_{0}\right)\right\},\left\{\psi_{j}\left(z_{0}\right)\right\}$ are orthonormal bases in $H_{z_{0}}$. Letting $\varphi_{j}=\phi\left(z_{0}\right) \varphi_{j}\left(z_{0}\right), \psi_{j}=\phi\left(z_{0}\right) \psi_{j}\left(z_{0}\right), \varphi_{j}(z)=\phi^{-1}(z) \varphi_{j}, \psi_{j}(z)=$ $\phi^{-1}(z) \psi_{j}, B \equiv \sum_{j=1}^{n} s_{j}(A)^{p\left(z_{0}\right)}\left\langle\cdot, \varphi_{j}\right\rangle_{H} \psi_{j}$, and $a(z)$ as before, we can define

$$
F(z) \equiv \phi^{-1}(z) B \phi(z)=\sum_{j=1}^{n} s_{j}(A)^{p\left(z_{0}\right) a(z)}\left\langle\cdot, \varphi_{j}(z)\right\rangle_{z} \psi_{j}(z),
$$

which is analytic, and $F\left(z_{0}\right)=A$. Moreover,

$$
\left\|F\left(e^{i \vartheta}\right)\right\|_{S_{p\left(e^{i \vartheta}\right)}\left(H_{\vartheta}\right)}=\left(\sum_{j=1}^{n} s_{j}(A)^{p\left(z_{0}\right)}\right)^{1 / p(\vartheta)}=1 .
$$

This implies that the norm of $A$ in the space $\left\{S_{p\left(e^{i \vartheta}\right)}\left(H_{\vartheta}\right)\right\}[z]$ is not larger than 1 . Using a density argument, we obtain the proof.

4. Factorization of operators. The following problem is of relevant interest in prediction theory: given a function $M$ on $\partial D$, we want to factor it as product of particular factors defined on $D$.

For scalar-valued functions this goes back to a famous theorem of G. Szegö [Sz]:

THEOREM 4.1. Let $M: \partial D \rightarrow[0, \infty]$ be measurable; then $M\left(e^{i \vartheta}\right)=\overline{\phi\left(e^{i \vartheta}\right)} \phi\left(e^{i \vartheta}\right)$ a.e. on $\partial D$, where $\phi \in H^{2}(D)$, if and only if $M$ and $\log M$ are integrable. The solution is unique if we require

and it is given by

$$
\phi(0)=\exp \int_{T} \log M\left(e^{i \vartheta}\right) \frac{d \vartheta}{2 \pi}
$$

$$
\phi(z)=\exp \frac{1}{2} \int_{T} \log M\left(e^{i \vartheta}\right) h_{z}(\vartheta) d \vartheta .
$$

The multidimensional version of the problem requries us to factor a positive definite, $n \times n$ matrix-valued function $M$ as $\phi^{*}\left(e^{i \vartheta}\right) \phi\left(e^{i \vartheta}\right)$, where $\phi(z)$ is invertible in $D$ and has entries in $H^{2}(D)$. The solution to this problem was obtained by $\mathrm{N}$. Wiener and P. Masani [WiMa] and, following a different approach, by H. Helson and D. Lowdenslager [HeLo]. A third, different proof can be found in [CCRSW 1], as a corollary of the interpolation results for families of finite-dimensional Banach spaces.

The infinite-dimensional version of the problem, where $M$ takes values in the set of positive, invertible, bounded operators acting on a separable Hilbert space $H$, has been solved by A. Devinatz [Dev] under the assumptions that $\|M\|$ and $\|\log M\|$ are integrable.

The factorization result that we obtain (Theorem $2.4(\mathrm{a}),(\mathrm{b}),(\mathrm{c})$ ) is an equivalent statement of Devinatz' theorem, under weaker assumptions.

\section{REFERENCES}

[CCRSW1] R. Coifman, M. Cwikel, R. Rochberg, Y. Sagher and G. Weiss, The complex method for interpolation of operators acting on families of Banach spaces, Lecture Notes in Math, vol. 779, Springer, 1980, pp. 125-153.

[CCRSW2] — A theory of complex interpolation for families of Banach spaces, Adv. in Math. 33 (1982), 203-229. 
[Dev] A. Devinatz, The factorization of operator valued functions, Ann. of Math. (2) 73 (1961), 458-495.

[GK] I. C. Gohberg and M. G. Krein, Introduction to the theory of linear non-self-adjoint operators in Hilbert spaces, Transl. Math. Monographs, vol. 18, Amer. Math. Soc., Providence, R.I., 1969.

[HeLo] H. Helson and D. Lowdenslager, Prediction theory and Fourier series in several variables, Part I, Acta Math. 99 (1959), 165-202; Part II, Acta Math. 106 (1962), 175-213.

[Hof] K. Hoffman, Banach spaces of analytic functions, Prentice-Hall, Englewood Cliffs, N.J., 1962.

[Sz] G. Szegö, Beitrage zur Theorie der Toeplizen Formen (Ersten Mitteilung), Math. Z. 6 (1920).

[Ves] E. Vesentini, On the subharmonicity of the spectral radius, Boll. Un. Mat. Ital. 4 (1968), 427-429.

[Vi] M. Vignati, Ph.D. Dissertation, Washington Univ., 1986.

[WiMa] N. Wiener and P. Masani, The prediction theory of multivariate stochastic processes, Part I, Acta Math. 98 (1957), 111-150; Part II, Acta Math. 99 (1957), 185-204.

Department of Mathematics, Siena College, Loudonville, New York 12211 (Current address of Steven Bloom)

Department of MAthematics, Washington University, St. LOUis, Missouri 63130

Current address (A. T. Vignati and Marco Vignati): Politecnico di Torino, 10129, Torino, Italy 